\title{
Congenital Disorder of Deglycosylation
}

National Cancer Institute

\section{Source}

National Cancer Institute. Congenital Disorder of Deglycosylation. NCI Thesaurus. Code C126746.

A rare autosomal recessive inherited disorder caused by mutations in the NGLY1 gene. It is characterized by developmental delay, hypotonia, abnormal involuntary movements, poor tear production, microcephaly, intractable seizures, abnormal eye movements, and liver abnormalities. 